\title{
Potential mechanism for bilateral sensory effects after a unilateral erector spinae plane block
}

\author{
Abdelghafour Elkoundi, MD $(\mathbb{D} \cdot$ Bensghir Mustapha, PhD • Alae El Koraichi, PhD
}

Received: 22 December 2019/Revised: 23 December 2019/ Accepted: 16 January 2020/Published online: 22 January 2020

(C) Canadian Anesthesiologists' Society 2020

To the Editor,

We read with interest the recent letter of Dr. Altıparmak et al. ${ }^{1}$ regarding the potential mechanisms for bilateral sensory effects after unilateral erector spinae plane blockade (ESPB) in patients undergoing laparoscopic cholecystectomy. Their observations allude to possible effects on the extent of sensory blockade resulting from pneumoperitoneum and patient positioning.

Pneumoperitoneum during laparoscopic surgery has important effects on abdominal wall mechanics and on the topography of the abdominal muscle layers. Consequently, it might influence the spread of the local anesthetic (LA) deposited between the muscle layers in the abdominal wall. Altıparmak et al. mainly based their arguments on the study in fresh-frozen cadavers by Desmet et al. where they injected methylene blue dye to study whether a pneumoperitoneum influences the spread of LA during the midaxillary approach of the transverse abdominus plane (TAP) block. $^{2}$ Nevertheless, we question certain points that they address.

This letter is accompanied by a reply. Please see Can J Anesth 2020; 67: this issue.

A. Elkoundi, MD $(\bowtie) \cdot$ B. Mustapha, PhD

Department of Anesthesiology and Intensive Care, Military

Hospital Mohammed V, Mohammed V University, Rabat,

Morocco

e-mail: abd.el.koundi@gmail.com

A. E. Koraichi, $\mathrm{PhD}$

Department of Pediatric Anesthesiology, Children Hospital of

Rabat, Faculty of Medicine and Pharmacy of Rabat, Mohammed

V University, Rabat, Morocco
Although Desmet et al. did not find a statistical difference in the methylene blue dyed surface area between the insufflated sides and non-insufflated sides, the percentage of stained segmental nerves was higher after insufflation. Altiparmak et al. conclude that, similarly to the results of Desmet et al., a pneumoperitonuem-related mechanism can influence the spread of LA solution during ESPB. Nevertheless, Desmet et al. clearly explained that a posterior spread was not possible during the TAP block and therefore excluded a spread toward the paravertebral space. The observed extension in their study was craniocaudal, in the region between the iliac crest and the costal margin, which explains that the percentage of stained segmental nerves was higher after insufflation; in no observations did the dye cross the midline.

The second question relates to the volume of LA used to perform the unilateral ESPB. The authors stated that 30-40 $\mathrm{mL}$ of $0.25 \%$ bupivacaine was injected unilaterally. We suggest that a volume of $40 \mathrm{~mL}$ is unusually large and may have unduly influenced the wide spread of the anesthetic more than the pneumoperitoneum did. Thus, it makes it difficult to know if the same effects would be expected with the much smaller volumes typically used in clinical practice.

Conflicts of interest None.

Funding statement None.

Editorial responsibility This submission was handled by Dr. Hilary P. Grocott, Editor-in-Chief, Canadian Journal of Anesthesia. 


\section{References}

1. Altıparmak B, Korkmaz, Toker M, Uysal Aİ. Potential mechanism for bilateral sensory effects after unilateral erector spinae plane blockade in patients undergoing laparoscopic cholecystectomy. Can J Anesth 2020; 67: 161-2.

2. Desmet M, Helsloot D, Vereecke E, Missant C, van de Velde M. Pneumoperitoneum does not influence spread of local anesthetics in midaxillary approach transversus abdominis plane block: a descriptive cadaver study. Reg Anesth Pain Med 2015; 40: 349-54.

Publisher's Note Springer Nature remains neutral with regard to jurisdictional claims in published maps and institutional affiliations. 